PROCEEDINGS OF THE

AMERICAN MATHEMATICAL SOCIETY

Volume 126, Number 10, October 1998, Pages 3065-3069

S 0002-9939(98)04360-3

\title{
EIGENVALUE PINCHING THEOREMS ON COMPACT SYMMETRIC SPACES
}

\author{
YUUICHI SUZUKI AND HAJIME URAKAWA \\ (Communicated by Christopher Croke)
}

\begin{abstract}
We prove two first eigenvalue pinching theorems for Riemannian symmetric spaces (Theorems 1 and 2). As their application, we answer negatively a question raised by Elworthy and Rosenberg, who proposed to show that for every compact simple Lie group $G$ with a bi-invariant Riemannian metric $h$ on $G$ with respect to $-\frac{1}{2} B, B$ being the Killing form of the Lie algebra $\mathfrak{g}$, the first eigenvalue $\lambda_{1}(h)$ would satisfy

$$
\sum_{j=1}^{2} \sum_{\ell=3}^{n}\left|\left[v_{j}, v_{\ell}\right]\right|^{2}>n\left(2 \lambda_{1}(h)-1\right),
$$

for all orthonormal bases $\left\{v_{j}\right\}_{j=1}^{n}$ of tangent spaces of $G$ (cf. Corollary 3 ). This problem arose in an attempt to give a spectral geometric proof that $\pi_{2}(G)=0$ for a Lie group $G$.
\end{abstract}

\section{§1. Eigenvalue Pinching theOREMS}

Lichnerowicz and Obata's celebrated theorem says (cf. [2]) that if the Ricci curvature of an $n$-dimensional compact Riemannian manifold $(M, g)$ satisfies

$$
\operatorname{Ric}_{g} \geq n-1,
$$

then the first eigenvalue $\lambda_{1}(g)$ of the Laplacian $\Delta_{g}$ satisfies

$$
\lambda_{1}(g) \geq n,
$$

and equality holds if and only if $(M, g)$ is the standard unit sphere $\left(S^{n}, g_{0}\right)$ of curvature 1.

It is known (cf. [8], [7], [3], [1]) that the following eigenvalue pinching theorem holds:

Theorem. Let $(M, g)$ be a compact $n$-dimensional Riemannian manifold with sectional curvature $K_{g} \geq 1$. Then, there exists a constant $C(n)>1$, depending only on $n$, such that, if $C(n) n \geq \lambda_{1}(g) \geq n, M$ is homeomorphic to $S^{n}$.

It would be a difficult problem to determine such a constant $C(n)$ explicitly. We would propose the conjecture that

$$
C(n) \geq\left(\frac{4}{n}+1\right) \frac{n-1}{n}>1 .
$$

Received by the editors November 21, 1996 and, in revised form, February 10, 1997.

1991 Mathematics Subject Classification. Primary 53C20.

Key words and phrases. First eigenvalue, pinching theorems, symmetric spaces.

(C)1998 American Mathematical Society 
Indeed, we prove under a strong symmetry condition,

Theorem 1. Let $(M, g)$ be a compact $n$-dimensional Riemannian manifold with Ricci curvature

$$
\operatorname{Ric}_{g} \geq n-1 \text {. }
$$

Assume that

$$
\left(\frac{4}{n}+1\right)(n-1) \geq \lambda_{1}(g) \geq n
$$

and $(M, g)$ is a simply connected, irreducible, Riemannian symmetric space. Then $(M, g)$ is the standard sphere $\left(S^{n}, g_{0}\right)$.

Proof. All simply connected compact irreducible Riemannian symmetric spaces are divided into two classes: type I or type II (cf. [5]). The spaces of type II are of the form $G / K$ with a compact simple Lie group $G$ and some closed subgroup $K$, and the invariant metric is given by $g=\frac{1}{2(n-1)} h$, where $h$ is a $G$-invariant Riemannian metric on $M=G / K$ induced from the Killing form of the Lie algebra $\mathfrak{g}$ of $G$. Then the Ricci tensor $\mathrm{Ric}_{h}$ satisfies

$$
\operatorname{Ric}_{h}=\frac{1}{2} h .
$$

All the first eigenvalues of $(M, h)$ are listed in the tables in [9]. A space of type I is a compact simply connected simple Lie group $G$, and $g=\frac{1}{4(n-1)} g^{\prime}$. Here $g^{\prime}$ is a bi-invariant metric on $G$ induced from the Killing form of $\mathfrak{g}$ whose Ricci tensor satisfies

$$
\operatorname{Ric}_{g^{\prime}}=\frac{1}{4} g^{\prime},
$$

and one can also find in [9] a list of the first eigenvalues of $\left(G, g^{\prime}\right)$. Due to these tables, one can see that all the first eigenvalues of $(M, g)$, except when $(M, g)$ is the unit sphere $\left(S^{n}, g_{0}\right)$, are bigger than $\left(\frac{4}{n}+1\right)(n-1)$.

\section{§2. ISOMETRIC IMMERSIONS}

In [6], Lawson and Simons proved the following theorem.

Theorem. Let $(M, g)$ be an $n$-dimensional compact Riemannian manifold isometrically immersed in the unit sphere $S^{N}(1)$ with second fundamental form $\beta$. Assume that

$$
\sum_{j=1}^{q} \sum_{\ell=q+1}^{n}\left(2\left|\beta\left(v_{j}, v_{\ell}\right)\right|^{2}-\left\langle\beta\left(v_{j}, v_{j}\right), \beta\left(v_{\ell}, v_{\ell}\right)\right\rangle\right)<q(n-q),
$$

for every orthonormal base $\left\{v_{j}\right\}_{j=1}^{n}$ of $T_{x} M(x \in M)$. Then the homology groups of $M$ vanish, $H_{q}(M, \mathbf{Z})=H_{n-q}(M, \mathbf{Z})=0$.

In [4], Elworthy and Rosenberg proved

Theorem. Let $(M, g)$ be an n-dimensional compact Riemannian manifold isometrically minimally immersed in $S^{N}(1)$. Assume that

$$
\sum_{j=1}^{q} \sum_{\ell=q+1}^{n} K_{g}\left(v_{j}, v_{\ell}\right)>\frac{q(n-q)}{2}
$$


for every orthonormal base $\left\{v_{j}\right\}_{j=1}^{n}$ of $T_{x} M, x \in M$. Then

$$
H_{q}(M, \mathbf{Z})=H_{n-q}(M, \mathbf{Z})=0 .
$$

In particular, if $K_{g}\left(v_{j}, v_{\ell}\right)>\frac{1}{2}$ for all $v_{j}, v_{\ell} \in T_{x} M(x \in M), j<\ell$, then $M$ is a homotopy sphere. Here $K_{g}(X, Y)$, for an orthonormal pair $X, Y \in T_{x} M$, means the sectional curvature of $(M, g)$ for a plane in $T_{x} M$ defined by $X$ and $Y$.

We show:

Theorem 2. Let $(M, h)$ be a compact simply connected irreducible Riemannian symmetric space and $h$ an invariant Riemannian metric induced from the Killing form of the Lie algebra of the isometry group of $(M, h)$. Assume that

$$
\sum_{j=1}^{2} \sum_{\ell=3}^{n} K_{h}\left(v_{j}, v_{\ell}\right)>\frac{n}{4}\left(2 \lambda_{1}(h)-1\right),
$$

for all orthonormal bases $\left\{v_{j}\right\}_{j=1}^{n}$ of $T_{x} M, x \in M$. Then $(M, h)$ is the unit sphere $\left(S^{n}, 2(n-1) g_{0}\right)$ of constant curvature $\frac{1}{2(n-1)}$.

Proof. In general, for a Riemannian manifold $(M, g)$,

$$
\begin{aligned}
\sum_{j=1}^{2} \sum_{\ell=3}^{n} K_{g}\left(v_{j}, v_{\ell}\right) & =\sum_{j=1}^{2} \sum_{\ell=1}^{n} K_{g}\left(v_{j}, v_{\ell}\right)-\sum_{j, \ell=1}^{2} K_{g}\left(v_{j}, v_{\ell}\right) \\
& =\operatorname{Ric}_{g}\left(v_{1}\right)+\operatorname{Ric}_{g}\left(v_{2}\right)-2 K_{g}\left(v_{1}, v_{2}\right) \\
& \geq 2\left(\inf \operatorname{Ric}_{g}-\max K_{g}\right) .
\end{aligned}
$$

For our case, since $(M, h)$ satisfies $\operatorname{Ric}_{h}=\frac{1}{2} h$,

$$
\sum_{j=1}^{2} \sum_{\ell=3}^{n} K_{h}\left(v_{j}, v_{\ell}\right)=1-2 K_{h}\left(v_{1}, v_{2}\right)
$$

which yields

$$
0 \leq \inf \sum_{j=1}^{2} \sum_{\ell=3}^{n} K_{h}\left(v_{j}, v_{\ell}\right)=1-2 \max K_{h} \leq 1 .
$$

Thus, the inequality (\#) implies $1 \geq \frac{n}{4}\left(2 \lambda_{1}(h)-1\right)$.

But, if we put $g=\frac{1}{2(n-1)} h$, then $\operatorname{Ric}_{g}=(n-1) g$, and the first eigenvalue $\lambda_{1}(g)$ of $g$ satisfies

$$
\left(\frac{4}{n}+1\right)(n-1) \geq \lambda_{1}(g) .
$$

Therefore, we have Theorem 2, because of Theorem 1 .

Theorem 2 immediately yields

Corollary 3. Let $G$ be a compact simply connected simple Lie group, and $h$ a biinvariant Riemannian metric $G$ induced from the Killing form $B$ of the Lie algebra $\mathfrak{g}$ of $G$ by identifying $G \cong G \times G / \Delta G$ as a symmetric space. That is,

$$
h_{x}\left(X_{x}, Y_{x}\right)=-\frac{1}{2} B(X, Y), \quad X, Y \in \mathfrak{g},
$$


where $X_{x}, Y_{x} \in T_{x} G, x \in G$. Assume that

$$
\sum_{j=1}^{2} \sum_{\ell=3}^{n}\left|\left[v_{j}, v_{\ell}\right]\right|^{2}>n\left(2 \lambda_{1}(h)-1\right),
$$

for every orthonormal base $\left\{v_{j}\right\}_{j=1}^{n}$ of $\mathfrak{g}$ with respect to $-\frac{1}{2} B$. Then $(M, h)$ is $(S U(2), h)$.

Remark 4. (1) In [4, pp. 74-75], Elworthy and Rosenberg proposed to give a spectral geometric proof that $\pi_{2}(G)=0$ for a Lie group $G$. The problem has been reduced to a question as to whether the above inequality in Corollary 3 would hold for all compact simply connected simple Lie group. But our Corollary 3 answers their question negatively.

(2) Let $(M, h)$ be a compact simply connected irreducible Riemannian symmetric space. Let

$$
\Phi_{1}:\left(M, \frac{\lambda_{1}}{n} h\right) \rightarrow S^{N}(1)
$$

be an isometric minimal immersion into the unit sphere defined by the first eigenfunctons of $(M, h)$. Let $\beta$ be the second fundamental form and $\widetilde{h}=\frac{\lambda_{1}}{n} h$. The inequality (\#) in Theorem 2 is equivalent to

$$
\sum_{j=1}^{2} \sum_{\ell=3}^{n} K_{\widetilde{h}}\left(v_{j}^{\prime}, v_{\ell}^{\prime}\right)>\frac{|\beta|^{2}}{2}+\frac{n}{2},
$$

for every orthonormal base $\left\{v_{j}^{\prime}\right\}_{j=1}^{n}$ of $T_{x} M(x \in M)$ relative to $\widetilde{h}$.

(3) Note that

$$
\max K_{h}=\max \left\{|\alpha|^{2} ; \alpha \in \Sigma^{+}(G, K)\right\},
$$

where $\Sigma^{+}(G, K)$ is the set of all positive roots $\alpha$ of $G$ relative to a Cartan subalgebra $\mathfrak{t}$ of $\mathfrak{g}$ whose restrictions $\left.\alpha\right|_{\mathfrak{a}}$ to $\mathfrak{a}$ do not vanish. Here $\mathfrak{g}=\mathfrak{k} \oplus \mathfrak{m}$ is the Cartan decomposition of $\mathfrak{g}$, and the Cartan subalgebra $\mathfrak{t}$ is by definition a maximal abelian subalgebra of $\mathfrak{g}$ containing a maximal abelian subalgebra $\mathfrak{a}$ of $\mathfrak{g}$ contained in $\mathfrak{m}$.

(4) If a Riemannian symmetric space $(M, g)$ satisfies the inequality (\#), then there might exist a $q$-th moment stable equation for $q \geq \frac{\operatorname{dim} M}{2}$ (cf. [4, p. 59]).

\section{REFERENCES}

[1] P. Berard, G. Besson and S. Gallot, Sur une inégalité isopérimétrique qui généralise celle de Paul Lévy-Gromov, Invent. Math. 80 (1985), 295-308. MR 86j:58017

[2] M. Berger, P. Gauduchon and E. Mazet, Le spectre d'une variété riemannienne, Lecture Notes in Math., Springer 194 (1971). MR 43:8025

[3] C.B. Croke, An eigenvalue pinching theorem, Invent. Math. 68 (1982), 253-256. MR 84a: 58084

[4] K.D. Elworthy and S. Rosenberg, Homotopy and homology vanishing theorems and the stability of stochastic flows, Geometric And Functional Analysis 6 (1996), 51-78. MR 97a:58198

[5] S. Helgason, Differential Geometry and Symmetric Spaces, Academic Press, 1962. MR 26:2986

[6] H.B. Lawson and J. Simons, On stable currents and their applications to global problems, Ann. of Math. 98 (1973), 427-450. MR 48:2881

[7] P. Li and A.E. Treiberg, Pinching theorem for the first eigenvalue on positively curved fourmanifolds, Invent. Math. 66 (1982), 35-38. MR 83i:53063 
[8] and J.Q. Zhong, Pinching theorem for the first eigenvalue on positively curved manifolds, Invent. Math. 65 (1981), 221-225. MR 83e:53044

[9] H. Urakawa, The first eigenvalue of the Laplacian for a positively curved homogeneous Riemannian manifold, Compositio Math. 59 (1986), 57-71. MR 87i:58174

Mathematics Laboratories, Graduate School of Information Sciences, Tohoku UniVersity, Katahira, SEndai, 980-8577, Japan

E-mail address: urakawa@math.is.tohoku.ac.jp 